\title{
Simulating Low-Level Laser Propagation From Skin Surface to Lumbar Disc, Knee, Femur and Prostate Gland By Monte Carlo Method
}

\author{
Trinh Tran Hong Duyen ${ }^{\mathrm{a}^{*}}$, Tran Anh Tu ${ }^{\mathrm{b}^{*}}$ \\ ${ }^{a, b}$ Ho Chi Minh City University of Technology, Vietnam National Ho Chi Minh City; 268 Ly Thuong Kiet street, \\ District 10, Ho Chi Minh city, postal code: 72506, Vietnam \\ ${ }^{a}$ Email: tt_hd2005@hcmut.edu.vn; ${ }^{b}$ Email:tranatu@hcmut.edu.vn
}

\begin{abstract}
Nowadays, the uses of laser and optics in the medical areas are extremely vivid, especially low-level laser therapy. The light with the wavelength of $633 \mathrm{~nm}$ to $1200 \mathrm{~nm}$ could penetrate and propagate deep in biological tissue. To develop the low-level laser therapy device, optimizing light delivery is critical to accurately stimulate the biological effects inside the biological tissue. Nevertheless, each form of the tissues at each zone on the body had various refractive optic, absorption, scattering, and anisotropy coefficients. This paper describes the simulation results of low-level laser propagation from skin surface at the lower spine, the knee, the femur and the prostate gland with four wavelengths $(633 \mathrm{~nm}, 780 \mathrm{~nm}, 850 \mathrm{~nm}$, and $940 \mathrm{~nm})$ by the Monte Carlo method. These simulation results are the base for developing the low-level laser therapy device, that could be used in clinical for treating the fracture, knee osteoarthritis, spinal degeneration, and benign prostatic hypertrophy.
\end{abstract}

Keywords: Monte Carlo simulation; propagation; wavelength; low-level laser therapy LLLT.

\section{Introduction}

The low-level laser therapy (LLLT) is getting more consideration and applying widely in the medical arena. This application promotes tissue regeneration, reduces inflammation and relieves pain. Unlike other medical laser applications, LLLT does not have an ablative or thermal mechanism, but rather a photochemical effect which means the light is absorbed and causes the photochemical reactions inside the biological tissue.

* Corresponding author. 
The reason why the technique is termed low level is that the optimum levels of energy density delivered are low and it is not comparable to other forms of laser therapy as practiced for ablation, cutting, and thermal tissue coagulation. The degree of photochemical reactions and the biological responses depends on the absorbed dose of light in biological tissue. The estimated absorbed dose within tissue requires understanding and studying thoroughly. The radiative transport equation (RTE) $)^{\mathrm{a}}$ is the popular equation for describing particle propagation in the complex structure of tissues [1]. One of the effective methods for solving the RTE equation is the simulation of propagation light in a scattering environment by the Monte Carlo method. This spread method has improved and applied in simulating the propagation of light in biological tissue, especially in the calculation and optimizing light delivery for low-level laser treatment [2-6]. This paper presents the establishment of the model and the propagation of low-level laser from the skin surface to the lumbar disc, knee, femur and prostate gland by Monte Carlo method. This simulation is based on the popular and prestigious Monte Carlo method for simulating light propagation in multi-layered tissue, the anatomical structure parameters in the human body, as well as the optical parameters of the biological tissues. These simulation results show the distribution of power density $^{\mathrm{b}}$ in the range $\left(1.0-10^{-4} \mathrm{~W} / \mathrm{cm}^{2}\right)$ of specific wavelengths $(633 \mathrm{~nm}, 780 \mathrm{~nm}, 850 \mathrm{~nm}$, and $940 \mathrm{~nm})$, respectively. These results show the "effective operating area" of the low-level laser beam on the tissue being treated. Thereby allowing selected wavelength, optical power and the appropriate dosage for treatment.

\section{Materials and Methods}

\subsection{Monte Carlo method}

The main base of modeling the light propagation simulation in biological tissues in this paper is the Monte Carlo method [7]. The Monte Carlo method is applied to simulate the propagation of light in biological tissue based on the RTE equation and the simulation calculation, computation of photon propagation in absorption and scattering medium [4-6, 8-11]. A photon is a unit of light. The wave properties of a photon are ignored, this paper only deals with particle properties. Therefore, the phase parameter and the polarization of light are not mentioned. In the simple case, the photon is put into an independent environment and its movement is recorded until it is absorbed or scattered out of the observation area. Although the results are highly accurate, this method requires repeated calculations many times to achieve the desired accuracy, resulting in a lot of time to perform the simulation. For example, to achieve an accuracy of $1 \%$, the movement of 10,000 photons must be recorded. A classic simulation method described by Prahl [3] and programmed by Wang and Jacques [11]. This method uses the technique of capturing hidden photons, known as "existing weights". Without stopping for each photon that will scatter until it is finished by an absorption process, these photons are given an "initial weight" and gradually decrease with each move at each scattering position. This improves the efficiency of the statistical method, which avoids the computational process of the photons moves many steps to make it just ended in an absorption process.

\footnotetext{
a Radiative transport equation (RTE) : $\frac{1}{v} \frac{\partial}{\partial \mathrm{t}} \mathrm{I}(\mathrm{r}, \mathrm{t}, \hat{\mathrm{s}})+\hat{\mathrm{s}} . \nabla \mathrm{L}(\mathrm{r}, \mathrm{t}, \hat{\mathrm{s}})=-\left(\mu_{\mathrm{a}}+\mu_{\mathrm{t}}\right) \mathrm{I}(\mathrm{r}, \mathrm{t}, \hat{\mathrm{s}})+\mu_{\mathrm{s}} \int_{4 \pi} \mathrm{p}\left(\hat{\mathrm{s}}, \hat{\mathrm{s}}^{\prime}\right) \mathrm{d}^{2} \hat{\mathrm{s}}^{\prime}+\varepsilon(\mathrm{r}, \mathrm{t}, \hat{\mathrm{s}})$

$\mathrm{b}$ Power density at which the laser beam can cause biological effects on tissue stimulation.
} 


\subsection{Parameters}

Parameters are structures of tissues, optical properties and scattering functions of tissues ${ }^{\mathrm{c}}$. These parameters are assumed to be homogeneous in the infinitesimal volume of biological tissue. The laser beam is assumed to be an extremely narrow beam, Gaussian distribution, exposure perpendicular to a laminated biological tissue sample as Figure 1.
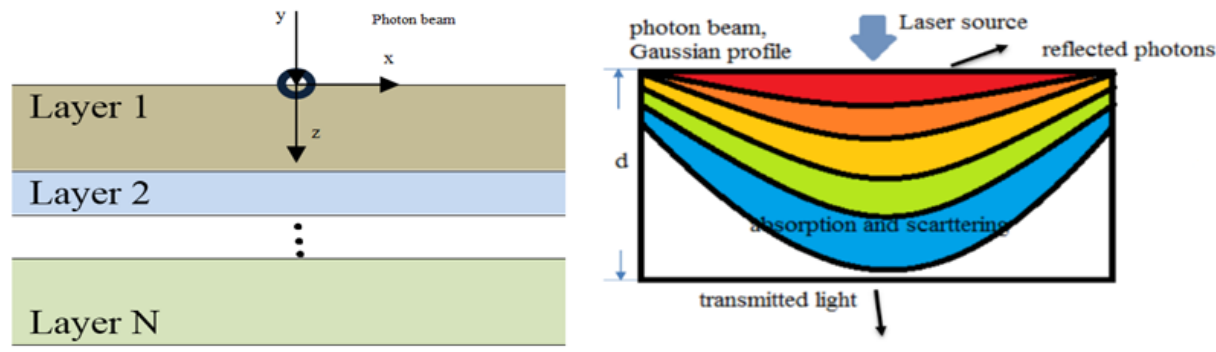

Figure 1: Model of class structure and photon scattering [11, 12]

These layers of tissue are considered parallel to each other, infinitely broad and characterized by structural and optical parameters. Although tissue never really wide to infinity, it can be regarded as extremely broad in terms of comparing the spatial distribution of the photons.

\subsection{Simulation models}

The structure of the human skin consists of stratum-corneum, epidermis, dermis with an approximate thickness $(0.06-0.1 \mathrm{~cm}),(0.0006-0.015 \mathrm{~cm}),(0.06-0.3 \mathrm{~cm})[13,14]$. In this paper, we use the modeling of general skin with an approximate thickness of $0.3 \mathrm{~cm}$. The first modeling structure of lumbar disc from skin consists of skin $\sim 0.3 \mathrm{~cm}$; fat $\sim 1.0 \mathrm{~cm}$; muscle $\sim 1.0 \mathrm{~cm}$; the lumbar spine. The second modeling structure of the knee from the skin at lateral patellar facets consists of skin $\sim 0.2 \mathrm{~cm}$; fat $\sim 0.9 \mathrm{~cm}$; muscle $\sim 0.7 \mathrm{~cm}$; synovial fluid. The third modeling structure of the lateral femur from skin consists of skin: $\sim 0.3 \mathrm{~cm}$; fat $\sim 1.2 \mathrm{~cm}$; muscle $\sim 2.0 \mathrm{~cm}$; femur. The fourth modeling structure of the prostate gland of the skin: skin $\sim 0.2 \mathrm{~cm}$; fat $\sim 0.2 \mathrm{~cm}$; muscle $\sim 1.6$ $\mathrm{cm}$; prostate gland. The absorption coefficient $\mu_{\mathrm{a}}$ and the scattering coefficient $\mu_{\mathrm{s}}$ are probability density functions, their inverse can be explained as the average mean distance for absorption and scattering. The total attenuation coefficient $\mu_{\mathrm{t}}$ is the sum of the absorption coefficient $\mu_{\mathrm{a}}$ and scattering coefficient $\mu_{\mathrm{s}}$, characterize the interaction of the photon average per unit of path length. Anisotropy coefficient $\mathrm{g}$, the value of the cosine of the angle average deviation scattering $\theta$ - the angle between the direction of the photons being scattered and incident photons, characterize isotropic properties of the medium. All of these parameters characterize the properties of each tissue layer and are referenced from reputable international publications and are presented in Tables 1.

c Thickness $d(\mathrm{~cm})$, absorption coefficient $\mu_{a}\left(\mathrm{~cm}^{-1}\right)$, scattering coefficient $\mu_{\mathrm{s}}\left(\mathrm{cm}^{-1}\right)$, total attenuation coefficient $\mu_{t}$ $\left(\mathrm{cm}^{-1}\right)$, refractive index $n$ and anisotropic coefficient $g$. 
The simulation results presented below are $0.1 \%$ accurate and calculated with 1,000,000 photons based on the improved Monte Carlo Multi-Layered (MCML) program [11] and the companion program called CONV [19] to enhance the performance and shorten simulation. Laser power will change to suit the position needed to achieve desired results with the continuous mode, Gaussian-shaped spatial beam profile (radius $1 / \mathrm{e}^{2}$ is $0.15 \mathrm{~cm}$ ).

Table 1: The optical parameters of the tissues

\begin{tabular}{|c|c|c|c|c|c|}
\hline Tissue & $\begin{array}{l}\lambda \\
(\mathrm{nm})\end{array}$ & $\mathbf{n}$ & $\begin{array}{l}\boldsymbol{\mu}_{\mathbf{a}} \\
\left(\mathrm{cm}^{-1}\right)\end{array}$ & $\begin{array}{l}\boldsymbol{\mu}_{\mathrm{s}} \\
\left(\mathrm{cm}^{-1}\right)\end{array}$ & g \\
\hline \multirow{4}{*}{ Skin [15-17] } & 633 & \multirow{4}{*}{1.4} & 0.334 & 272.9 & 0.9 \\
\hline & 780 & & 0.142 & 197.3 & 0.9 \\
\hline & 850 & & 0.1223 & 175.73 & 0.9 \\
\hline & 940 & & 0.1905 & 156.7 & 0.9 \\
\hline \multirow{4}{*}{$\begin{array}{l}\text { Subcutaneous fat } \\
{[18]}\end{array}$} & 633 & \multirow{4}{*}{1.44} & 0.128 & 125.5 & 0.9 \\
\hline & 780 & & 0.0846 & 114.67 & 0.9 \\
\hline & 850 & & 0.086 & 110.9 & 0.9 \\
\hline & 940 & & 0.168 & 108.6 & 0.9 \\
\hline \multirow{4}{*}{ Muscle [18] } & 633 & \multirow{4}{*}{1.37} & 1.32 & 89.6 & 0.9 \\
\hline & 780 & & 0.331 & 71.2 & 0.9 \\
\hline & 850 & & 0.295 & 66.0 & 0.9 \\
\hline & 940 & & 0.401 & 58.1 & 0.9 \\
\hline
\end{tabular}

\section{Results}

To measure the light density distributed along the $\mathrm{x}$-axis, we divide the reflected and transmitted edge into $1 \mathrm{~cm}$ intervals. After the input data file is prepared, the program MCML can be executed using the input data file "file.mci". When the calculation is completed, the result will be written into the output data file as the named in the input data file - "file.mco". We used the MATLAB programming language which is used to launch the file ".m” to show each result of MCML - "file.mco". It only works for individual results of MCML - "file.mco". These simulation results show the distribution of power density in $10^{-4} \mathrm{~W} / \mathrm{cm}^{2}$ - at which the laser beam can cause the stimulation biological effect on tissue. The simulation results of the spreading of $633 \mathrm{~nm}$ wavelength reach a depth smaller than the remaining wavelengths. It can be observed through its distribution image. The program Conv is created to handling the output data file of $\mathrm{Mcml}$ that responses of infinitely narrow photon beam, and makes the output data if the responses of finite size beam are to be computed. Almost initial beams propagate along the y-axis, then disperse slowly at a different orientation. After the program is invoked, the menu system will direct the data input, output, or process - "file.iso". We used the Kaleida Graph software that produces publication-quality graphs and fit data with arbitrary curves. We can combine the distribution results at $10^{-4} \mathrm{~W} / \mathrm{cm}^{2}$ and make them smooth. Figure 2 shows the simulation results which are distributed at $10^{-4} \mathrm{~W} / \mathrm{cm}^{2}$ of power density from the skin surface to the lumbar disc. The total energy of the Gaussian laser beam is $10 \mathrm{~J}$. The penetrating ability into the lumbar disc area of $780 \mathrm{~nm}$ and $850 \mathrm{~nm}$ wavelengths are so depth $(\sim 6.0 \mathrm{~cm})$ than that of $940 \mathrm{~nm}$ wavelength $(\sim 5.0 \mathrm{~cm})-$ Figure $2 \mathrm{a}$. The $633 \mathrm{~nm}$ wavelength is less penetrating tissue $(\sim 3.5 \mathrm{~cm})$ than that of other wavelengths - Figure $2 b$.

For knee joint position, the thickness at lateral patellar facets into the synovial fluid is thin. We have calculated the total energy of the Gaussian laser beam is $10 \mathrm{~J}$. The penetrating ability into the tissue of $780 \mathrm{~nm}, 850 \mathrm{~nm}$, and $940 \mathrm{~nm}$ wavelengths are quite similar $(\sim 4.5 \mathrm{~cm})$ - Figure $3 \mathrm{a}$. The $633 \mathrm{~nm}$ wavelength is less penetrating than 
that of other wavelengths into tissue $(\sim 2.0 \mathrm{~cm})$ - Figure $3 \mathrm{~b}$.

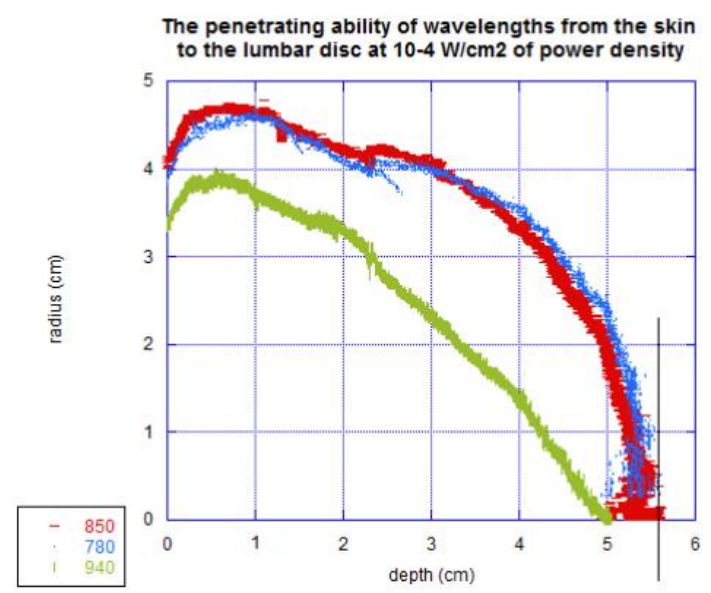

a) $780 \mathrm{~nm}, 850 \mathrm{~nm}$, and $940 \mathrm{~nm}$

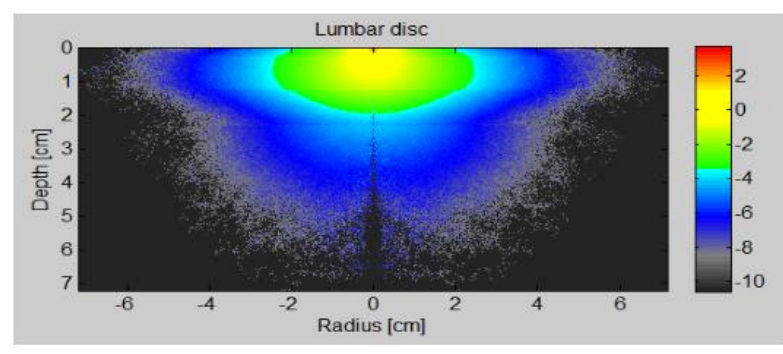

b) $633 \mathrm{~nm}$

Figure 2: The penetrating ability of wavelengths $(633 \mathrm{~nm}, 780 \mathrm{~nm}, 850 \mathrm{~nm}$, and $940 \mathrm{~nm})$ from the skin to the lumbar disc at $10^{-4} \mathrm{~W} / \mathrm{cm}^{2}$ of power density.

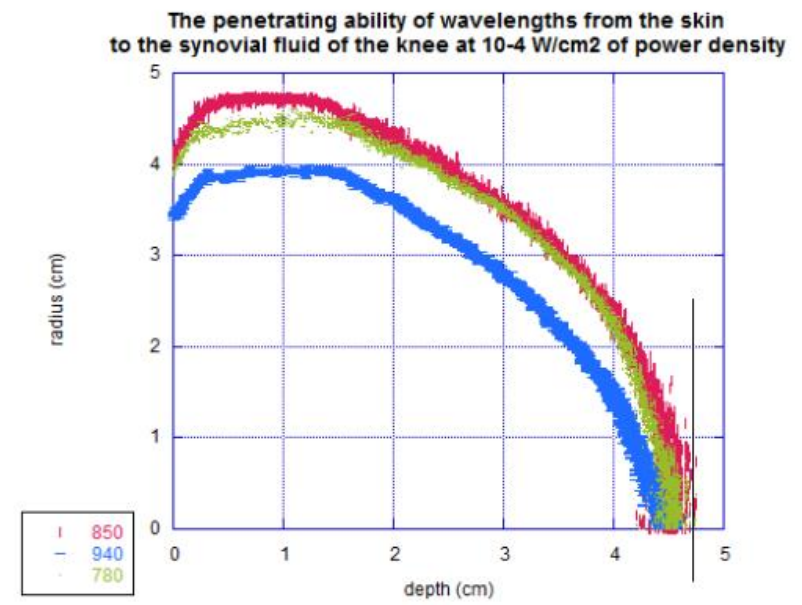

a) $780 \mathrm{~nm}, 850 \mathrm{~nm}$, and $940 \mathrm{~nm}$

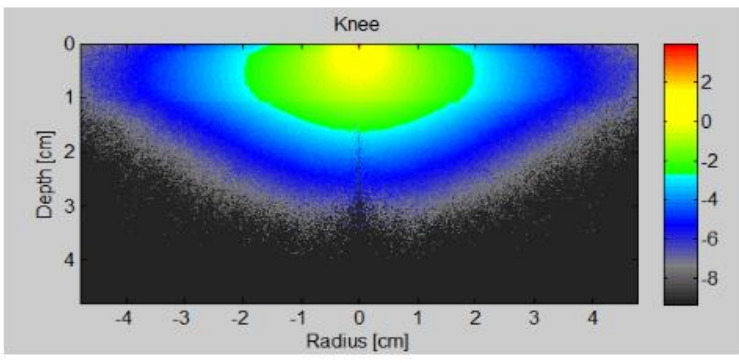

b) $633 \mathrm{~nm}$

Figure 3: The penetrating ability of wavelengths $(633 \mathrm{~nm}, 780 \mathrm{~nm}, 850 \mathrm{~nm}$, and $940 \mathrm{~nm})$ from the skin to the synovial fluid of the knee at $10^{-4} \mathrm{~W} / \mathrm{cm}^{2}$ of power density.

Figure 4 shows the simulation result from the skin surface to the lateral femur. The total energy of the Gaussian laser beam is $10 \mathrm{~J}$. The penetrating ability into the tissue of $780 \mathrm{~nm}, 850 \mathrm{~nm}$, and $940 \mathrm{~nm}$ wavelengths are quite similar $(\sim 5.2 \mathrm{~cm})$ - Figure 4a. The $633 \mathrm{~nm}$ wavelength is less penetrating than that of other wavelengths into tissue $(\sim 2.5 \mathrm{~cm})$ - Figure $4 \mathrm{~b}$. 


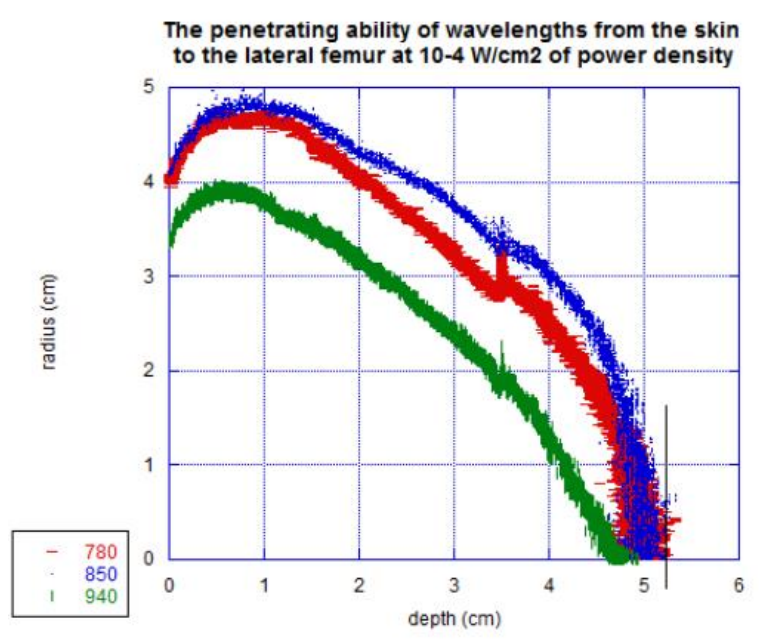

a) $780 \mathrm{~nm}, 850 \mathrm{~nm}$, and $940 \mathrm{~nm}$

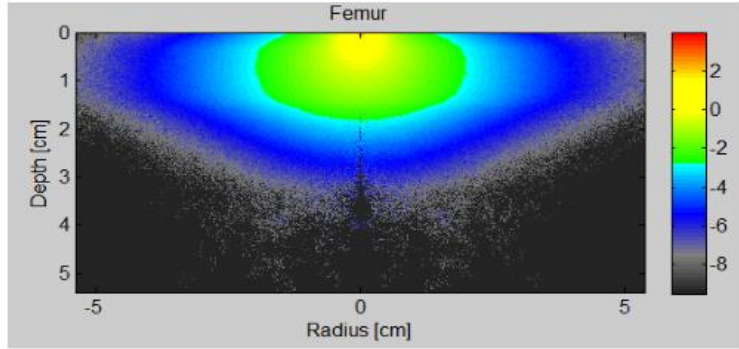

b) $633 \mathrm{~nm}$

Figure 4: The penetrating ability of wavelengths $(633 \mathrm{~nm}, 780 \mathrm{~nm}, 850 \mathrm{~nm}$, and $940 \mathrm{~nm})$ from the skin to the lateral femur at $10^{-4} \mathrm{~W} / \mathrm{cm}^{2}$ of power density.

Figure 5 shows the simulation result from the skin surface to the prostate gland with the total energy of the Gaussian laser beam is $10 \mathrm{~J}$. The total energy of the Gaussian laser beam is $10 \mathrm{~J}$. The penetrating ability into the lumbar disc area of $780 \mathrm{~nm}$ and $850 \mathrm{~nm}$ wavelengths are so depth $(\sim 5.5 \mathrm{~cm})$ than that of $940 \mathrm{~nm}$ wavelength $(\sim$ $5.0 \mathrm{~cm})$ - Figure $5 \mathrm{a}$. The $633 \mathrm{~nm}$ wavelength is less penetrating tissue $(\sim 3.5 \mathrm{~cm})$ than that of other wavelengths - Figure 5b.

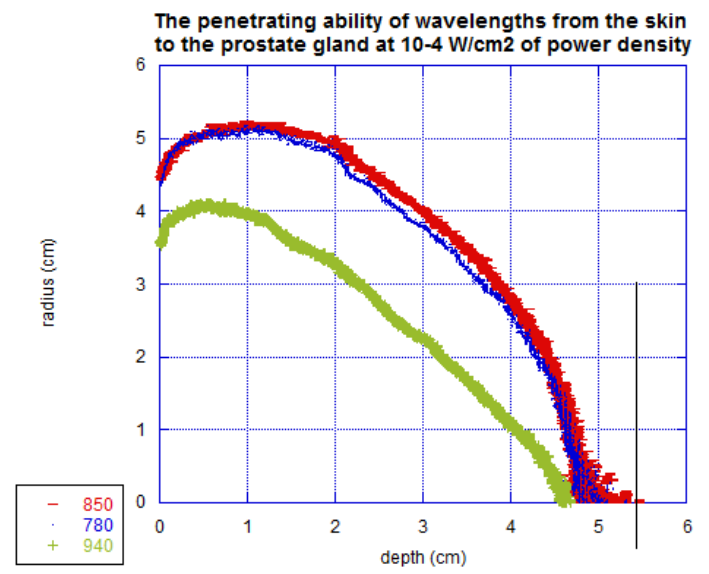

a) $780 \mathrm{~nm}, 850 \mathrm{~nm}$, and $940 \mathrm{~nm}$

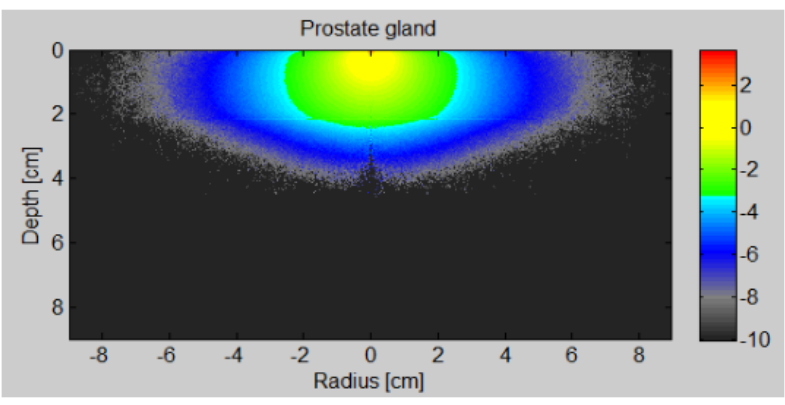

b) $633 \mathrm{~nm}$

Figure 5: The penetrating ability of wavelengths $(633 \mathrm{~nm}, 780 \mathrm{~nm}, 850 \mathrm{~nm}$, and $940 \mathrm{~nm})$ from the skin surface to the prostate gland at $10^{-4} \mathrm{~W} / \mathrm{cm}^{2}$ of power density.

On the other hand, when the irradiation time increases, the "depth of penetration" and "impact radius" of the beam also increase significantly. For the pelvic area (lumbar disc, knee, thigh), it is required as much energy of the beam as exposed to the deep (prostate gland). This is not entirely true for all cases of the body, because 
tissue structures are different in each region, thickness and each tissue has different characterizes.

\section{Conclusions}

Today, patients often face one or more different symptoms when they are sick. They may have only one or more diseases. Therefore, these types of devices can combine flexibly with each other to become a powerful tool to support the therapy for patients in the best way. The propagation of the laser beam into biological tissue was simulated by the Monte Carlo method. From the simulation results, the low-level laser of $780 \mathrm{~nm}, 850 \mathrm{~nm}$, and $940 \mathrm{~nm}$ wavelength can fully impact tissue layers from the skin surface to the lumbar spine, knee, femur and prostate gland. In case of a desire to provide light to the deeper tissues, to increase the strength to provide sufficient energy to the target tissue, near-infrared light can penetrate the soft tissue deep within the tissue, while the red light does not reach the depth when performed under the same conditions [20]. Although the spreading of $633 \mathrm{~nm}$ wavelength reaches a depth smaller than the remaining wavelengths. It can be used for skin treatment equipment or intravenous. The laser interacts with the component of blood, which makes improve microcirculation, balances the erythrocytes, hemoglobin concentration, reduces the cholesterol, rheological properties, and blood lipids [21,22]. These wavelengths are useful and suitable for developing the low-level laser therapy device. As the irradiation time increases, the "depth of penetration" and "impact radius" of the beam also increase. These simulation results allow further studies to apply low-level laser of $780 \mathrm{~nm}, 850 \mathrm{~nm}$, and $940 \mathrm{~nm}$ wavelengths in the low-level laser therapy non-invasively from the skin surface in the treatment of fracture, knee osteoarthritis, spinal degeneration, and benign prostatic hypertrophy.

\section{Acknowledgments}

All authors would like to thank the support of Dr.Eng. Tran Trung Nghia, Associate Dean of Faculty of Applied Science, Ho Chi Minh City University of Technology, Vietnam National Ho Chi Minh City for his supervision, suggestions, contributions, support as well as improve the content of this article.

\section{References}

[1]. S. Chandrasekhar, "Radiative transfer," ed. London: Oxford University Press, 1950.

[2]. B. C. Wilson and G. Adam, "A Monte Carlo model for the absorption and flux distributions of light in tissue," Medical Physics, vol. 10, pp. 824-830.

[3]. S. Prahl, M. Keijzer, S. Jacques, et al., "A Monte Carlo Model of Light Propagation in Tissue," SPIE Inst. Ser. IS, vol. 5, 01/01/1989.

[4]. S. T. Flock, M. S. Patterson, B. C. Wilson, et al., "Monte Carlo modeling of light propagation in highly scattering tissues. I. Model predictions and comparison with diffusion theory," IEEE Transactions on Biomedical Engineering, vol. 36, pp. 1162-1168, 12/1989.

[5]. S. T. Flock, B. C. Wilson, and M. S. Patterson, "Monte Carlo modeling of light propagation in highly scattering tissues. II. Comparison with measurements in phantoms," IEEE Transactions on Biomedical Engineering, vol. 36, pp. 1169-1173, 12/1989.

[6]. M. Keijzer, S. L. Jacques, S. A. Prahl, et al., "Light distributions in artery tissue: Monte Carlo 
simulations for finite-diameter laser beams," Lasers in Surgery and Medicine, vol. 9, pp. 148-154, 1989.

[7]. N. Metropolis and S. Ulam, "The Monte Carlo Method," Journal of the American Statistical Association, vol. 44, pp. 335-341, 01/09/1949.

[8]. M. Keijzer, J. W. Pickering, and M. J. C. van Gemert, "Laser beam diameter for port wine stain treatment," Lasers in Surgery and Medicine, vol. 11, pp. 601-605, 1991.

[9]. S. L. Jacques and L. Wang, "Monte Carlo Modeling of Light Transport in Tissues," in Optical-Thermal Response of Laser-Irradiated Tissue, A. J. Welch and M. J. C. Van Gemert, Eds., ed Boston, MA: Springer US, 1995, pp. 73-100.

[10]. L. Wang and S. L. Jacques, "Hybrid model of Monte Carlo simulation and diffusion theory for light reflectance by turbid media," Journal of the Optical Society of America A, vol. 10, pp. 1746-1752, 01/08/1993.

[11]. L. Wang, S. L. Jacques, and L. Zheng, "MCML-Monte Carlo modeling of light transport in multilayered tissues," Computer Methods and Programs in Biomedicine, vol. 47, pp. 131-146, 01/07/1995.

[12]. $\quad$ A. Abdo, A. Ersen, and M. Sahin, "Near-infrared light penetration profile in the rodent brain," Journal of biomedical optics, vol. 18, p. 75001, 01/07/2013.

[13]. B. D. Chaurasia, Human anatomy vol. 1. India: CBS Publishers \& Distributors Pvt Ltd, 1991.

[14]. G. F. Odland, "Structure of skin," in Physiology. Biochemistry and molecular biology of the skin, L. A. Goldsmith, Ed., ed New York: Oxford University Press, 1991, pp. 3-62.

[15]. J. Mobley, "Optical Properties of Tissue," in Biomedical Photonics Handbook, T. Vo-Dinh, Ed., ed: CRC Press, 2003.

[16]. R. R. Anderson and J. A. Parrish, "The Optics of Human Skin," Journal of Investigative Dermatology, vol. 77, pp. 13-19, 1981/07/01/.

[17]. J. M. Schmitt, G. X. Zhou, E. C. Walker, et al., "Multilayer model of photon diffusion in skin," Journal of the Optical Society of America A, vol. 7, pp. 2141-2153, 01/11/1990.

[18]. A. N. Bashkatov, E. A. Genina, V. I. Kochubey, et al., "Optical properties of human skin, subcutaneous and mucous tissues in the wavelength range from 400 to $2000 \mathrm{~nm}$," Journal of Physics D: Applied Physics, vol. 38, pp. 2543-2555, 22/07/2005.

[19]. L. Wang, S. L. Jacques, and L. Zheng, "Conv-convolution for responses to a finite diameter photon beam incident on multi-layered tissues," Computer Methods and Programs in Biomedicine, vol. 54, pp. $141-150,01 / 11 / 1997$.

[20]. J. Jagdeo, L. Adams, N. Brody, et al., "Transcranial Red and Near Infrared Light Transmission in a Cadaveric Model," PloS one, vol. 7, p. e47460, 15/10/2012.

[21]. V. Mikhaylov, "The use of Intravenous Laser Blood Irradiation (ILBI) at 630-640 nm to prevent vascular diseases and to increase life expectancy," Laser Therapy, vol. 24, pp. 15 - 26, 31/03/2015.

[22]. N. I. Nechipurenko, L. N. Anatskaia, L. I. Matusevich, et al., "Effect of intravenous laser irradiation on some blood biochemical indicators in the acute stage of lacunar infarcts," Zh Nevrol Psikhiatr Im S S Korsakova, vol. 114, pp. 43-8, 02/09/2014. 